\title{
Anti-inflammatory effect and mechanisms of Huangqi glycoprotein in treating experimental autoimmune encephalomyelitis
}

\author{
Huiyu Zhang ${ }^{1}$, Minfang Guo ${ }^{1}$, Lihong Zhang ${ }^{1}$, Huiqing Xue², Zhi Chai², Yuqing Yan ${ }^{1}$, Yanxia Xing ${ }^{1}$, Baoguo Xiao ${ }^{3}$, \\ Peijun Zhang ${ }^{1}$, Cungen Ma1,2 \\ ${ }^{1}$ Institute of Brain Science, Department of Neurology, Medical School, Shanxi Datong University, Datong, 2"2011" Collaborative \\ Innovation Center/Research Center of Neurobiology, Shanxi University of Traditional Chinese Medicine, Taiyuan, ${ }^{3}$ Institute of \\ Neurology, Huashan Hospital, Institutes of Brain Science and State Key Laboratory of Medical Neurobiology, Fudan University, \\ Shanghai, China
}

\begin{abstract}
Huangqi glycoprotein (HQGP) is prepared from Astragalus membranaceus by ammonium sulfate precipitation. It was indicated that HQGP has an immunoregulatory effect. In this study, we established a chronic experimental autoimmune encephalomyelitis (EAE) model and observed the therapeutic effect and possible mechanisms of HQGP (intraperitoneally at $1 \mathrm{mg} / \mathrm{kg} /$ day) on EAE. The results showed that HQGP delayed onset and ameliorated severity of EAE, and reduced the infiltration and accumulation of pathogenic $T$ cells in the central nerves system (CNS). HQGP also reduced the production of IL-6, IL-17 and TNF- $\alpha$ and increased the level of IL-10. However, the level of IFN- $\gamma$ production was also increased in HQGP-treated mice compared with EAE control mice. In brain, chemokines such as CCL2 and CCL5 were inhibited in HQGP-treated EAE compared with control mice. These results demonstrate that HQGP alleviates the pathogenesis of EAE possibly by suppressing the neuroinflammation and decreasing the secretion of chemokines and cell adhesion.
\end{abstract}

Key words: Huangqi glycoprotein, experimental autoimmune encephalomyelitis, inflammatory factor, chemokines, immunomodulation.

\section{Introduction}

Multiple sclerosis (MS) is an autoimmune inflammatory disease of the central nervous system (CNS). The pathological changes include infiltration of immune cells, demyelination and axonal loss in the CNS [8]. Experimental autoimmune encephalomyelitis (EAE), a well-established animal model of MS, exhibits similar pathological characteristics and clin- ical manifestations as human MS. In recent years, due to the rapid development of neuroimaging and molecular biology, the number of clinically diagnosed MS patients has been increasing. In contrast, there is no economical and effective treatment.

In the pathogenesis of MS and EAE, autoreactive $T$ cells are first activated in the periphery and migrated through the blood-brain barrier (BBB) into the CNS. 
Cytokines and chemokines play an important role in the inflammatory process by regulating the activation, proliferation and migration of immune cells. Meanwhile, the process of inflammation of EAE/MS is accompanied by increased levels glutamate and excitotoxicity. The detrimental effects of glutamate in EAE/MS pathogenesis was mediated by a large family of glutamate receptors [24]. The expression of chemokines is significantly correlated with disease severity $[3,4]$. Among them, CCL2 (MCP-1) plays a major role in the recruitment of monocytes in CNS lesions of MS [11]. In murine EAE, the expression of CCL2 in the brain and spinal cord was up-regulated and enhanced the proliferation and recruitment of $T$ cells into the CNS, which causes more pathological inflammation [10]. In the pathophysiology of EAE, CCL2 and CCL5 (RANTES) contribute to the regulation of the intracerebral adhesion of leukocytes [7].

Huangqi glycoprotein (HQGP) is one of the extracts of Astragalus membranaceus. It was indicated that HQGP has an immunoregulatory effect. Our previous studies showed that HQGP can delay onset and ameliorate severity of EAE, accompanied by reduced neuroinflammation of EAE via reducing the number of reactive $T$ cell subsets and inhibiting the secretion of inflammatory cytokines [25]. In this study, we aimed to further explore novel potential mechanism(s) of HQGP in suppressing the development of EAE mice.

\section{Material and methods \\ Animals}

Female C57BL/6 mice, 8-10 weeks old and with body weight of 18-20 g, were purchased from Vital River Laboratory Animal Technology Co. Ltd. (Beijing, China). All experiments were conducted in accordance with the guidelines of the International Council for Laboratory Animal Science. The study was approved by the Ethics Committee of Shanxi Datong University, Datong, China.

\section{Huangqi glycoprotein}

HQGP was provided by the experimental center of Shanxi University of Chinese Medicine. The main chemical components of Astragalus membranaceus were Astragalus polysaccharides, saponins, flavonoids, trace elements and amino acids. Astragalus membranaceus was extracted by Tris- $\mathrm{HCl}$ buffer. The crude extract was captured by anion exchange chromatography, and finely separated by hydrophobic chromatography and gel filtration chromatography. The result showed that the electrophoretically pure HQGP was obtained by the three-step purification of Q Sepharose Fast Flow, Butyl Sepharose High Performance and Superdex 75 10/300 GL from the crude extraction. The relative molecular weight of HQGP was $16.801 \mathrm{kDa}$.

\section{EAE induction and HQGP treatment}

Induction and assessment of EAE were performed as in our previous study [25]. Chronic EAE was induced by subcutaneous immunization of the upper dorsal flanks with $300 \mu \mathrm{g}$ of MOG $_{35-55}$ in Freund's complete adjuvant (Sigma, USA) supplemented with $3 \mathrm{mg} / \mathrm{ml}$ of Mycobacterium tuberculosis H37Ra (BD Difco, USA) (400 $\mu \mathrm{g} /$ mice). Mice were then injected with 700 ng of pertussis toxin (Enzo Life Sciences, USA) via the abdominal cavity at the same time of immunization and again $48 \mathrm{~h}$ later. Clinical assessment of EAE scores was evaluated daily using the international general scale of 0-5 as instructed [23].

HQGP dissolved in normal saline (NS) was injected intraperitoneally at $1 \mathrm{mg} / \mathrm{kg} /$ day on day 3 post-immunization (p.i.) until day 21 p.i. The injection of NS was set up as a control in a similar manner.

\section{Histology and immunohistochemistry}

On day 22 p.i., mice were perfused and fixed with saline and 4\% (w/v) buffered paraformaldehyde. The brains and spinal cords (lower thoracic-lumbar) were removed and sliced $(10 \mu \mathrm{m})$. The pathological changes were detected by hematoxylin/eosin ( $\mathrm{H} \& \mathrm{E})$ staining. For immunohistochemistry, non-specific binding was blocked with 3\% bovine serum (Serotec, UK) in $0.3 \%$ Triton X-100/PBS for 30 minutes at room temperature (RT). The sections were incubated with anti-CD4 (1 : 1000; eBioscience), anti-CD68 (1 : 1000; eBioscience), anti-CCL2 (1:500; Abcam UK), and anti-CCL5 (1: 500; Abcam UK) at $4^{\circ} \mathrm{C}$ overnight. The sections were incubated further with corresponding secondary antibodies at RT for $2 \mathrm{~h}$. The negative controls were treated similarly, but the primary antibodies were omitted.

\section{Cytokine ELISA}

On day 22 p.i., mice were sacrificed and spleens were removed under aseptic conditions. Suspension of mononuclear cells (MNCs) was prepared from spleen and cultured for $72 \mathrm{~h}$ at $37^{\circ} \mathrm{C}$ in a humidified 


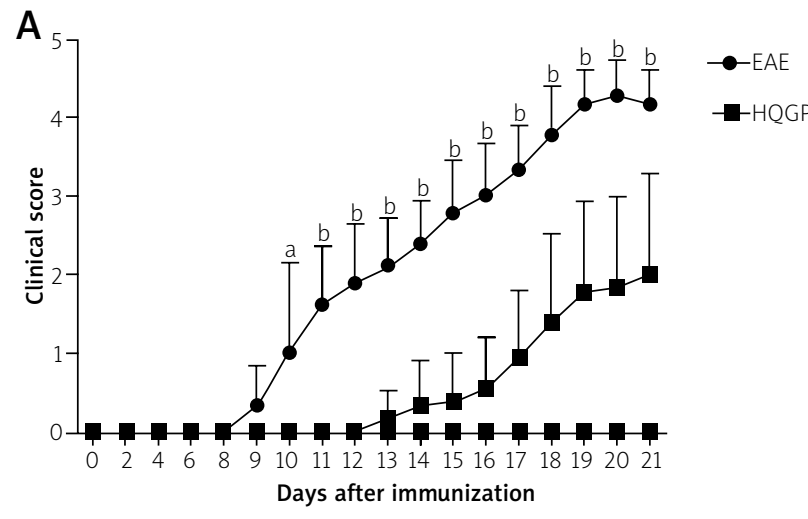

B

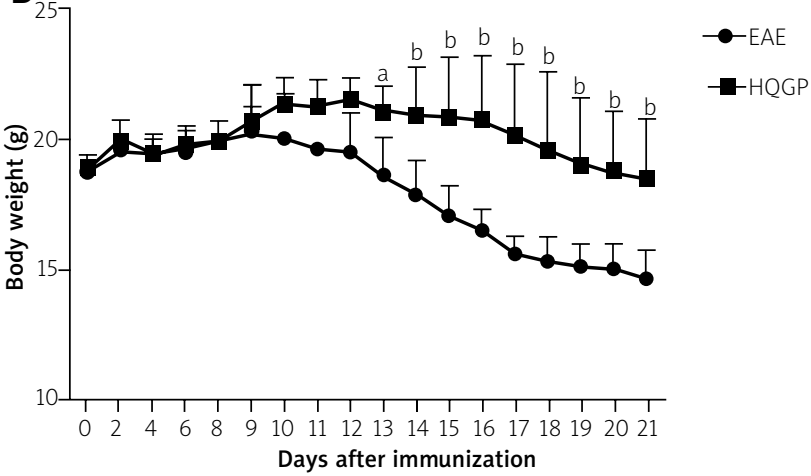

Fig. 1. Huangqi glycoprotein delayed onset and ameliorated severity of experimental autoimmune encephalomyelitis. A) Clinical score. B) Body weight. ${ }^{a} p<0.01 ;{ }^{b} p<0.001$.

atmosphere containing $5 \% \mathrm{CO}_{2}$. The supernatant of cells was collected, and levels of IL-6, TNF- $\alpha$, IFN- $\gamma$, IL-10 and IL-17 were measured using sandwich ELISA kits (R\&D Systems Inc) in accordance with the manufacturer's instructions. Determination was performed in duplicate in 3 independent experiments. The results were expressed as $\mathrm{pg} / \mathrm{ml}$.

\section{Western blot analysis}

Proteins from brains were extracted and protein concentrations were determined by a Bradford protein assay. Equal amounts of protein $(30 \mu \mathrm{g})$ were separated by SDS-PAGE, and transferred onto PVDF membrane (Millipore). Membranes were blocked with 5\% non-fat milk, and incubated with antiCCL2 (1: 500, Pepro tech Inc.), anti-CCL5 (1: 500, Pepro tech Inc.) and anti-GAPDH (1:1,000, Epitomics) overnight at $4^{\circ} \mathrm{C}$. After washing in TBST, the immunoblots were incubated with HRP-conjugated secondary antibodies (Thermo Scientific, MA, USA) at RT for $1 \mathrm{~h}$. Bands were visualized by a chemiluminescence (ECL) kit under an ECL system (Millipore).

\section{Statistical analysis}

All values were expressed as the mean $\pm S D$ and analyzed by GraphPad Prism software. A level of $p<0.05$ was considered statistically significant.

\section{Results \\ HQGP delays onset and ameliorates severity of EAE}

To observe the therapeutic effect of HQGP in EAE, HQGP was injected intraperitoneally on day 3 p.i. of EAE. The result showed that the incidence of EAE was $100 \%$ and there was no death. In the EAE group, the mean onset date was day $9.89 \pm$ 0.93 , and the mean maximum score was $4.28 \pm 0.15$. The HQGP treatment delayed onset $(15.22 \pm 2.54$, $p<0.001)$ and reduced the maximum clinical score $(2.00 \pm 0.42, p<0.001)$ (Table 1, Fig. 1).

\section{HQGP inhibits inflammation}

We evaluated the pathology of CNS inflammation. As shown in Figure $2 \mathrm{~A}$, mice with EAE showed extensive infiltration of immune cells in spinal cord (15.67 \pm 2.08). The HQGP treatment significantly inhibits infiltration of immune cells into the spinal cord compared with the EAE control $(6.33 \pm 1.53, p<0.01)$.

Immunohistochemistry shows extensive infiltration of inflammatory $\mathrm{CD} 4^{+} \mathrm{T}$ cells and $\mathrm{CD} 8^{+}$macrophages in spinal cords (Fig. 2B). The HQGP treatment obviously reduced the infiltration of CD4 T+ cells and CD68+ macrophages in spinal cords as compared with control EAE (Fig. 2B).

Table 1. Clinical score and body weight of experimental autoimmune encephalomyelitis mice

\begin{tabular}{ccccc}
\hline Group & $n$ & Incidence (\%) & Mean onset date (days) & Mean score of maximal symptoms \\
\hline EAE & 9 & 100 & $9.89 \pm 0.93$ & $4.28 \pm 0.15$ \\
\hline HQGP & 9 & 100 & $15.22 \pm 2.54^{\mathrm{a}}$ & $2.00 \pm 0.42^{\mathrm{a}}$ \\
\hline
\end{tabular}

$a_{p}<0.001$. Data are expressed as mean $\pm S D$

EAE - experimental autoimmune encephalomyelitis, HQGP - Huangai glycoprotein 
A
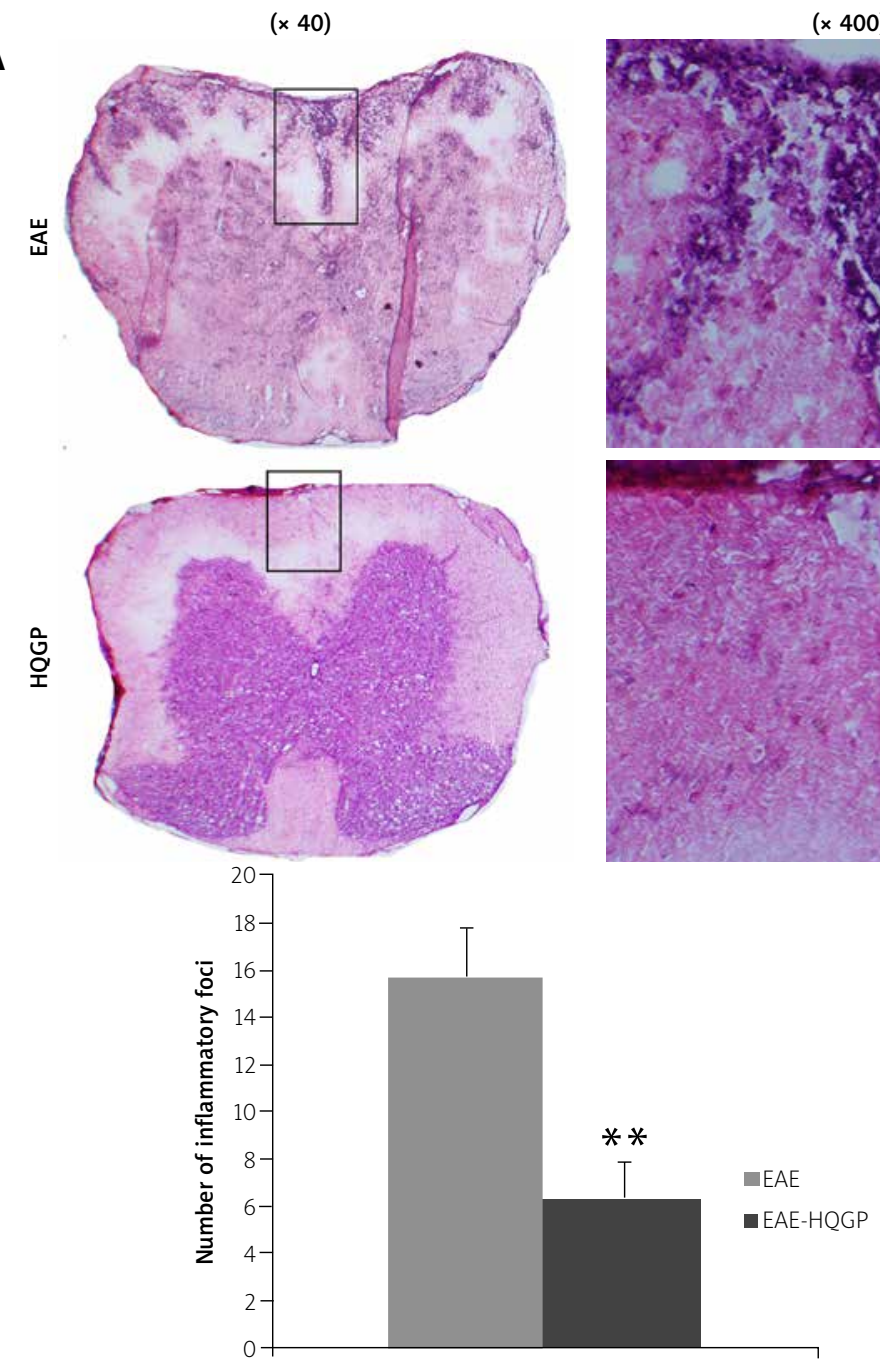

B
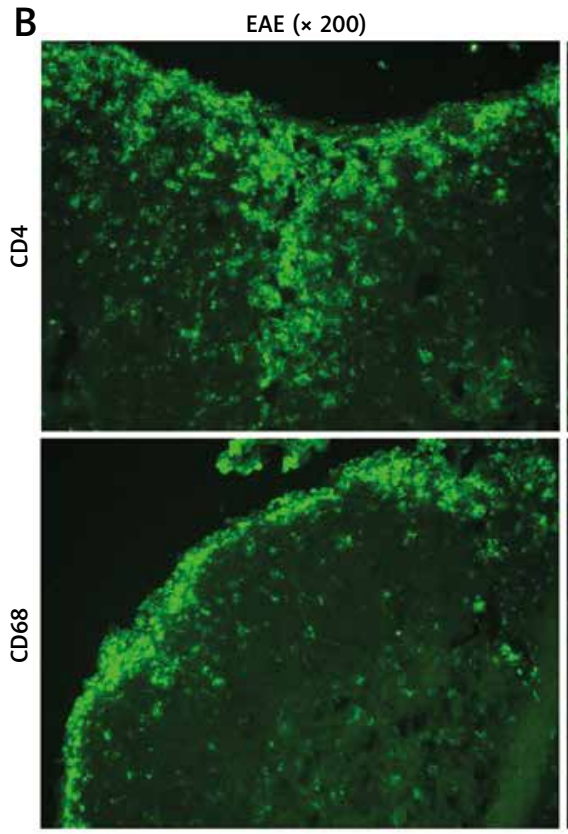

$\operatorname{HQGP}(\times 200)$
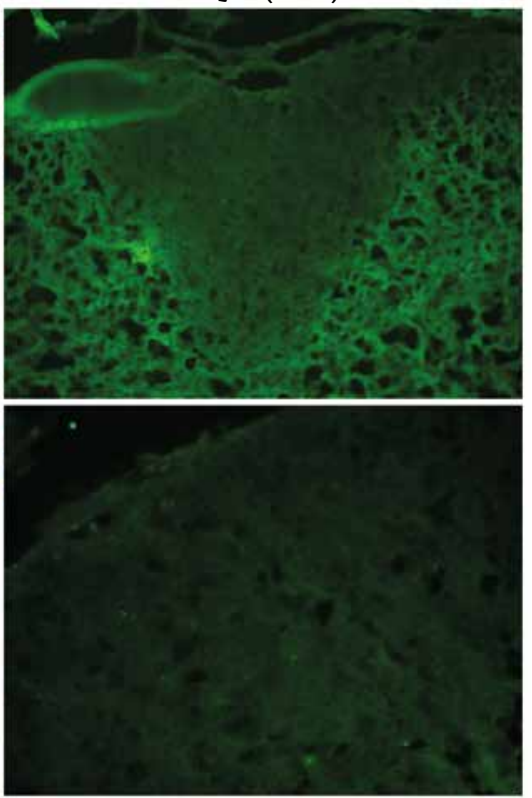

$(\times 400)$
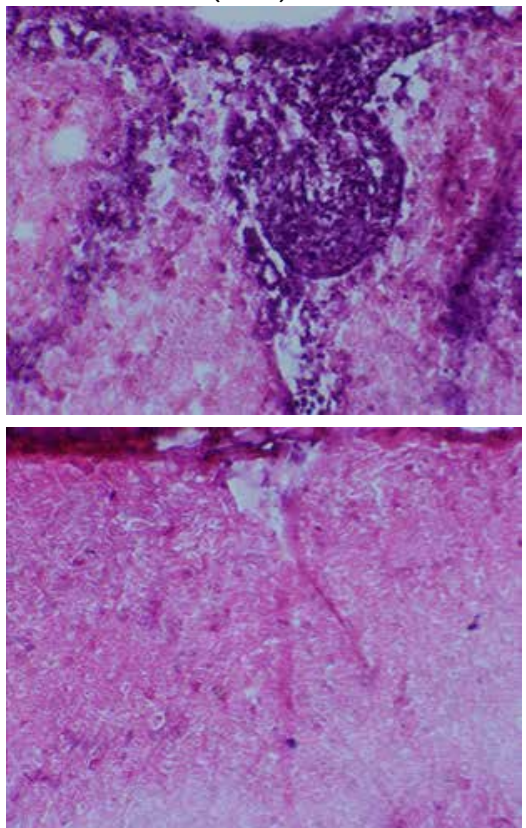

Fig. 2. A) Inflammation stained with H\&E stain. The number of inflammatory foci (> 20 mononuclear cells/ focus) in whole spinal cord was calculated by Image-Pro Plus software. Mice with experimental autoimmune encephalomyelitis (EAE) showed extensive infiltration of immune cells in spinal cord. When compared with the EAE control, Huangqi glycoprotein (HQGP)-treated mice had a significant improvement in the extent of inflammation in spinal cords $\left({ }^{\star \star} p<0.01\right)$. Representative patterns were obtained from one of three experiments with similar results. B) The infiltration of CD4+ $T$ cells and CD68+ macrophage in spinal cords. The infiltration of CD4+ $T$ cells and CD68+ macrophage was significantly lower in the HQGP-treated group compared with control EAE mice. Representative patterns were obtained from one of three experiments with similar results. 


\section{HQGP inhibits the inflammatory cytokines}

The increased number of infiltrating immune cells in the CNS would produce pro-inflammatory mediators to create an inflammatory microenvironment. In our study, we measured levels of cytokine IL-6, TNF- $\alpha$, IFN- $\gamma$, IL-10 and IL-17 in the supernatant of cultured splenic MNCs. As expected, levels of IL- 6 , IL-17 and TNF- $\alpha$ were significantly suppressed, and the level of IL-10 was significantly increased in mice treated with HQGP compared with control EAE mice (Fig. 3, $p<0.05$ ). However, the level of IFN- $\gamma$ production was also increased in HQGP-treated mice com-
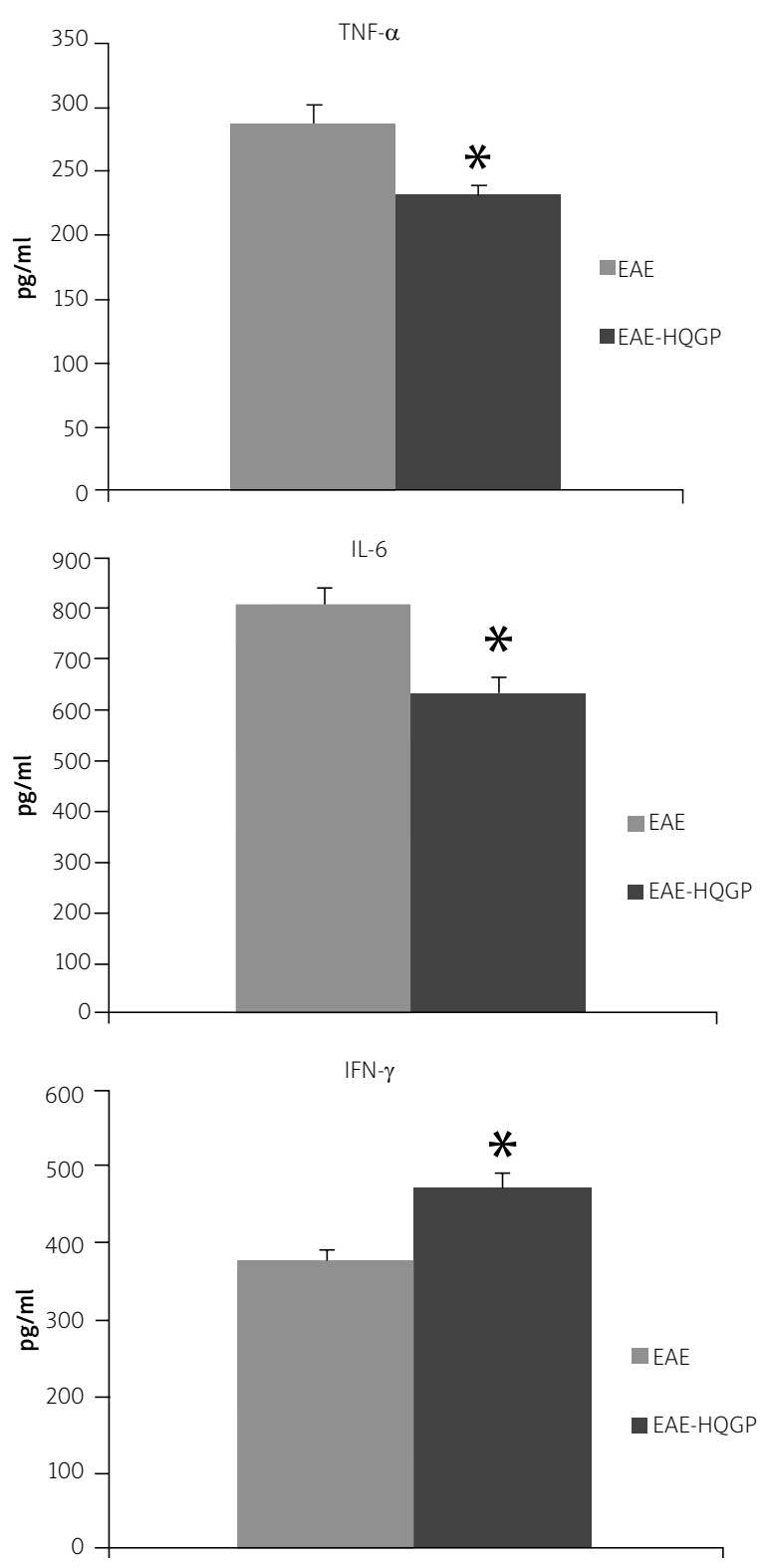

pared with EAE control mice $(p<0.05)$. These results indicate that HQGP alleviates the neuroinflammatory microenvironment of spinal cord by inhibiting the infiltration of immune cells.

\section{HQGP suppresses the expression of CCL2 and CCL5 protein in EAE mice}

To understand the mechanism of HQGP in inhibiting the migration of inflammatory cells into the CNS, we stained brain slices of EAE mice with antiCCL2 and anti-CCL5 antibodies. In EAE mice treated with HQGP, the expression of CCL2+ and CCL5 in brain was obviously reduced compared with saline
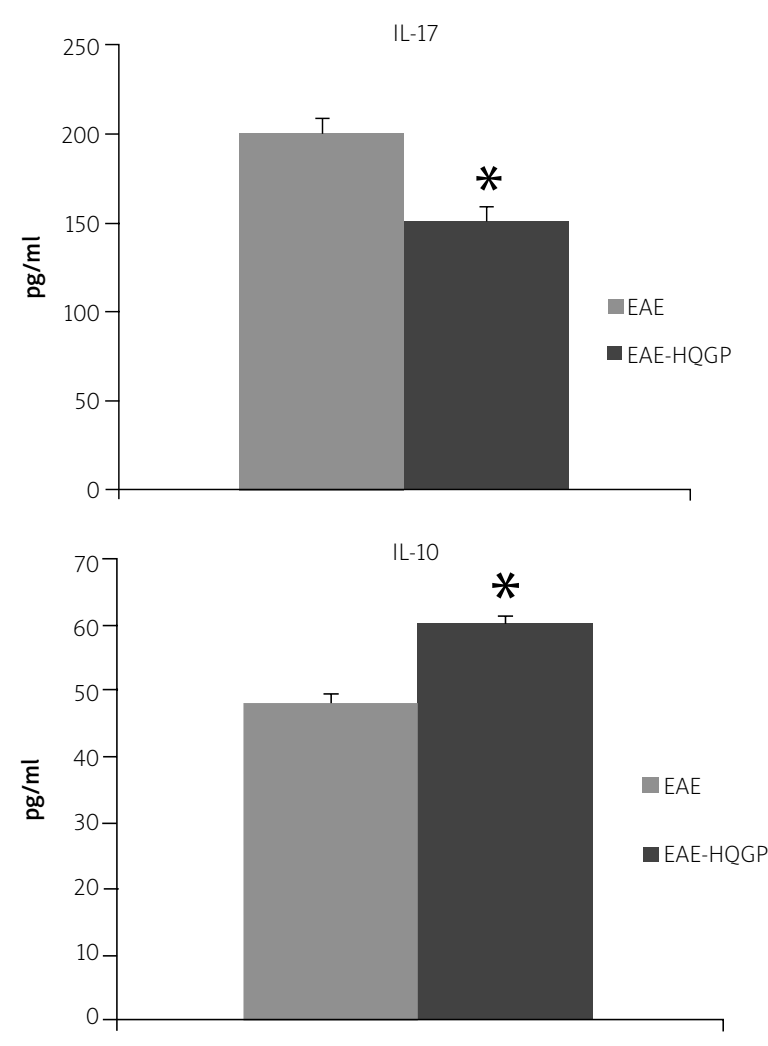

Fig. 3. Huangqi glycoprotein reduces inflammatory responses in the peripheral immunity. The supernatant of mononuclear cells of spleen was collected. Production of IL- 6 , TNF- $\alpha$, IFN- $\gamma$, IL-10 and IL-17 was measured using ELISA kits. ${ }^{*} p<0.05$. 

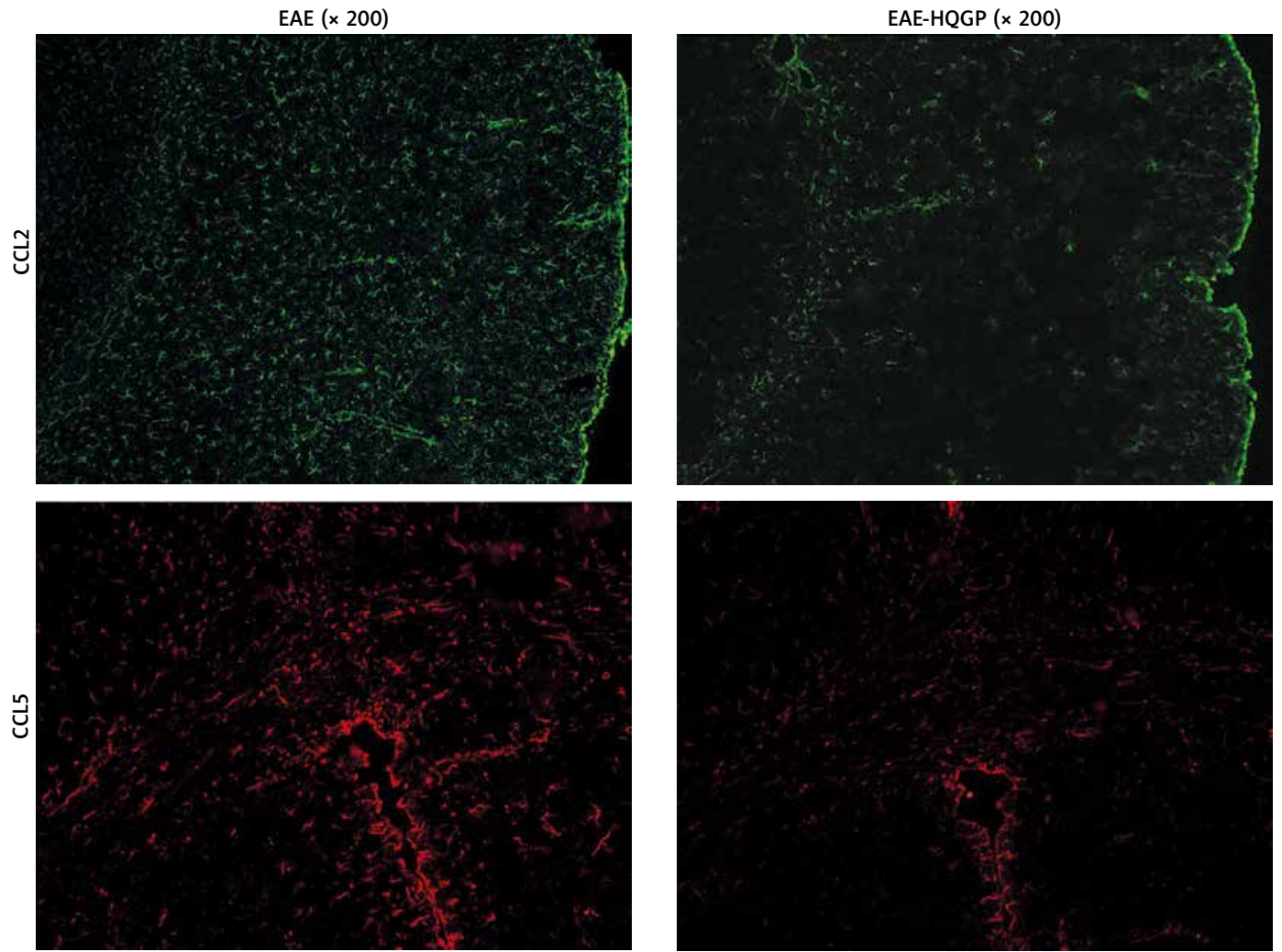

Fig. 4. Immunohistochemistry of CCL2 and CCL5 in brain of experimental autoimmune encephalomyelitis (EAE) mice. Chronic EAE was induced by subcutaneous immunization with MOG35-55, and injected with Huangqi glycoprotein (HQGP) on day 3 p.i. to 21 p.i. On day 21 p.i., mice were sacrificed and the slices of brain $(10 \mu \mathrm{m})$ were stained with anti-CCL2 and anti-CCL5 antibodies. Representative patterns were obtained from one of two experiments with similar results.

control mice (Fig. 4), suggesting that inhibition of CCL2 and CCL5 expression might reduce the infiltration of inflammatory cells into the CNS in EAE.

We further detected the expression of CCL2 and CCL5 protein derived from brain using Western blot techniques. As expected, the HQGP treatment decreased levels of CCL2 and CCL5 protein in brains compared with control EAE mice (Fig. 5, $p<0.05$ ). These data were in agreement with the immunohistochemistry results (Fig. 4).

\section{Discussion}

The therapies of western medicine have potential side-effects and high prices. Traditional Chinese medicine (TCM) can be used to treat the complex and varied presentations of MS, with few side-effects [13]. HQGP, an effective component extracted from Astragalus membranaceus had positive effects on neuroprotective and immune regulation in MS/EAE [25]. Long- term usage of HQGP has few side-effects and large potential benefits.

Our study showed that HQGP delayed the onset of EAE and ameliorated the severity of EAE. Consistent with clinical improvement, HQGP inhibited the migration of inflammatory cells into the spinal cord. The disruption of the blood brain barrier (BBB) and the migration of activated T-cells and macrophages across the BBB have a critical role in the occurrence and development of MS and EAE $[20,1]$. In the complex process of EAE, various cells of the immune system, including CD4+ Th1 and Th17, $\gamma \delta T$ cells, CD8+ $\mathrm{T}$ cells, Treg cells and macrophages, are involved [12]. The inflammatory infiltration in the CNS mainly consists of activated T cells and macrophages [16]. Immunofluorescence labeling indicated that the number of infiltrated CD4+ T cells and CD68+ macrophages also decreased after HQGP treatment. These data presented for the first time demonstrate that HQGP inhibits the infiltration of immune cells into 

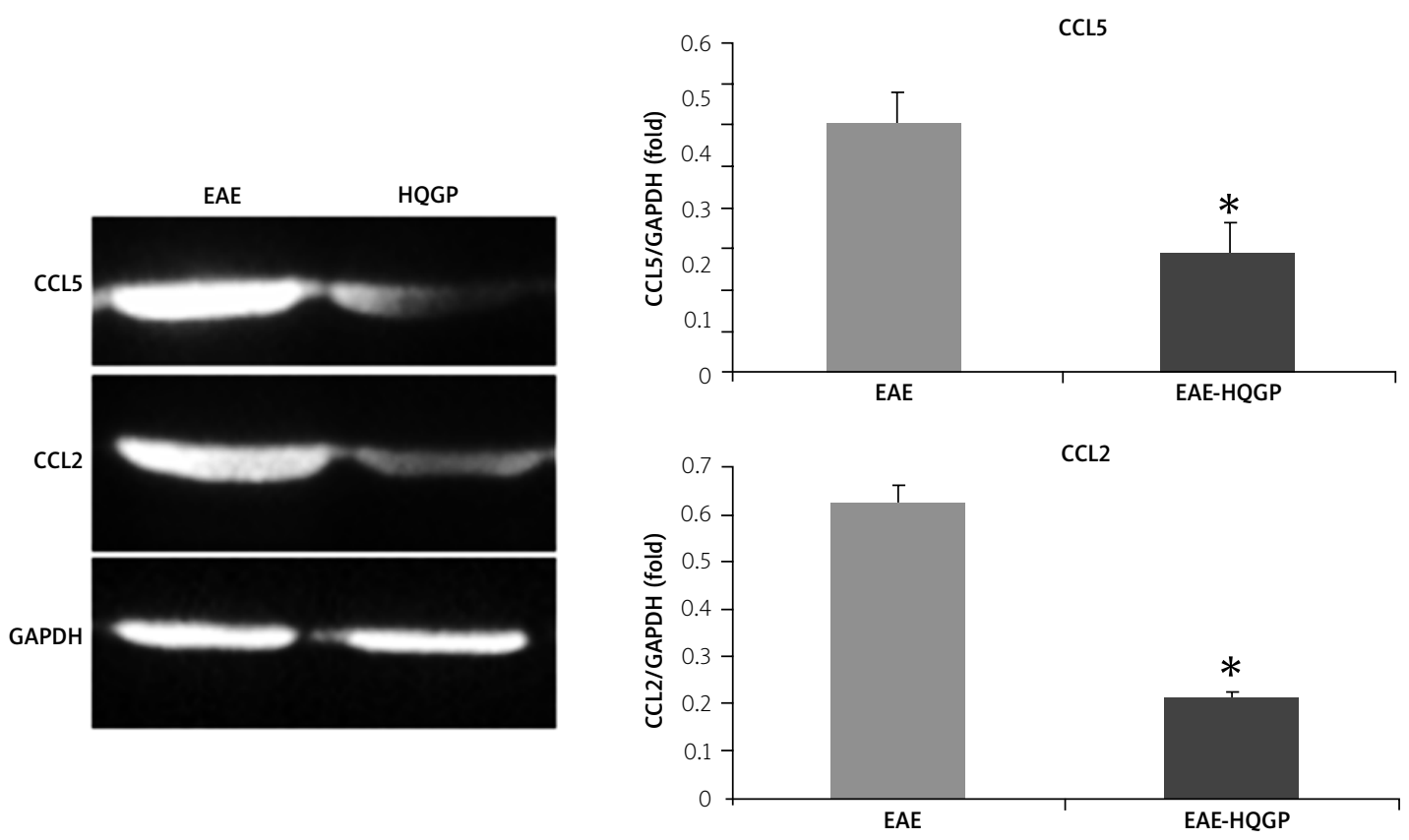

Fig. 5. The expression of CCL2 and CCL5 protein in brains was measured by Western blot. The results were represented as representative bands, and quantitative analysis was done from three independent experiments. ${ }^{*} p<0.05$.

the spinal cord, contributing to the reduction of the inflammatory microenvironment of the spinal cord and the improvement of the clinical score of EAE.

Th1 and Th17 cells release IFN- $\gamma$ and IL-17, and are thought to be the major inflammatory cells, while Th2 and Treg cells produce IL- 4 and IL-10, and are anti-inflammatory. TNF- $\alpha$, IL-1 $\beta$, and IL- 6 were the major inflammatory cytokines released by activated macrophages [19]. In our current study, HQGP suppressed EAE possibly by inhibiting the production of pro-inflammatory cytokines IL-6, TNF- $\alpha$ and IL-17 and promoting production of the immunoregulatory cytokine IL-10. Collectively, these data demonstrate an immunoregulatory effect of HQGP in the treatment of EAE. IFN- $\gamma$ is a multifunctional cytokine that is involved in the initiation and establishment of inflammation, and participates in both innate and adaptive immune responses. Although IFN- $\gamma$ was released by Th1 cells, the effect in EAE is questionable. It was reported that IFN- $\gamma$ ameliorates EAE by limiting myelin lipid peroxidation [21]. Loss of IFN- $\alpha$ enables the expansion of autoreactive $\mathrm{CD}^{+}{ }^{+} \mathrm{T}$ cells to induce experimental autoimmune encephalomyelitis [17]. Our results showed that HQGP increased the production of IFN- $\gamma$, accompanied by improvement of the clinical score of
EAE. It is possible that IFN- $\gamma$ acts as a disease-limiting agent within the CNS but not in peripheral immune tissues. These results suggest that HQGP may have a different mechanism of action compared with other immunosuppressive agents.

The chemokines expressed by inflammatory lesions in the CNS of MS enhanced recruitment of activated Th1 and Th17 cells and macrophages across the disrupted BBB. CCL2 was first identified as a potent chemotaxin for monocytes in response to pro-inflammatory stimuli [14]. CCL2 regulates the migration and activation of monocytes, T cells, NK cells and basophils and plays an important role in innate immunity [18]. The important role of CCL2 for Th1 immune responses in the pathogenesis of EAE has been reported [9]. Specifically, CCL2 production from resident CNS was thought to contribute to the recruitment of myeloid dendritic cells and macrophages during EAE [5]. A recent study showed that less favorable spinal cord expression of CCL2 could diminish infiltration of CD4+ T cells, including highly pathogenic IL-17 + IFN- $\gamma$ + cells, and inflammatory monocytes into the spinal cord [15]. The pathological overproduction of CCL5 is a hallmark of disease progression in MS [22]. A previous study suggested that 
induction of leukocyte adhesion to the brain microvasculature is an important mechanism by which CCL2 and CCL5 participate in the pathophysiology of EAE [6]. Recent data provide evidence that CCL5/ CCR5 might mediate the infiltration of type I T cells into the CNS during the disease development of EAE [2]. In our study, the HQGP treatment inhibited the expression of CCL2 and CCL5, which could decrease the infiltration of inflammatory cells in the CNS.

In conclusion, HQGP delays the onset and ameliorates the severity of EAE, accompanied by inhibition of the inflammatory responses in the CNS. HQGP suppresses CNS inflammatory responses possibly through inhibiting cytokine/chemokine secretion and cell adhesion. Further studies on the cellular and molecular mechanisms are still necessary.

\section{Acknowledgements}

Supported by: the National Natural Science Foundation of China, No. 81473577 the Natural Science Foundation of Shanxi Province, No. 2013011052-4. International S\&T Cooperation Program of China, No. 2013DFA30700. Science and technology key projects of Shanxi Province, No. 20130321031-31.

\section{Disclosure}

\section{Authors report no conflict of interest.}

\section{References}

1. Bennett J, Basivireddy J, Kollar A, Biron KE, Reickmann P, Jefferies WA, McQuaid S. Blood-brain barrier disruption and enhanced vascular permeability in the multiple sclerosis model EAE. J Neuroimmunol 2010; 229: 180-191.

2. Chen Q, Liu Y, Lu A, Ni K, Xiang Z, Wen K, Tu W. Influenza virus infection exacerbates experimental autoimmune encephalomyelitis disease by promoting type I T cells infiltration into central nervous system. J Autoimmun 2017; 77: 1-10.

3. Dargahi N, Katsara M, Tselios T, Androutsou ME, de Courten M, Matsoukas J, Apostolopoulos V. Multiple Sclerosis: Immunopathology and Treatment Update. Brain Sci 2017; 7. pii: E78; doi: 10.3390/brainsci7070078.

4. Dendrou CA, Fugger L, Friese MA. Immunopathology of multiple sclerosis. Rev Immunol 2015; 15: 545-558.

5. Dogan RN, Elhofy A, Karpus WJ. Production of CCL2 by central nervous system cells regulates development of murine experimental autoimmune encephalomyelitis through the recruitment of TNF- $\alpha$ and iNOS-expressing macrophages and myeloid dendritic cells. J Immunol 2008; 180: 7376-7384.

6. dos Santos AC, Barsante MM, Arantes RM, Bernard CC, Teixeira MM, Carvalho-Tavares J. CCL2 and CCL5 mediate leukocyte adhesion in experimental autoimmune encephalomyelitis - an intravital microscopy study. J Neuroimmunol 2005; 162: 122 129.

7. Dos Santos AC, Roffê E, Arantes RM, Juliano L, Pesquero JL, Pesquero JB, Bader M, Teixeira MM, Carvalho-Tavares J. Kinin B2 receptor regulates chemokines CCL2 and CCL5 expression and modulates leukocyte recruitment and pathology in experimental autoimmune encephalomyelitis (EAE) in mice. J Neuroinflammation 2008; 5: 49.

8. Fancy SP, Kotter MR, Harrington EP, Huang JK, Zhao C, Rowitch DH, Franklin RJ. Overcoming remyelination failure in multiple sclerosis and other myelin disorders. Exp Neurol 2010; 225: 18-23.

9. Huang DR, Wang J, Kivisakk P, Rollins BJ, Ransohoff RM. Absence of monocyte chemoattractant protein 1 in mice leads to decreased local macrophage recruitment and antigen-specific $T$ helper cell type 1 immune response in experimental autoimmune encephalomyelitis. J Exp Med 2001; 193: 713-726.

10. Ji Z, Fan Z, Zhang Y, Yu R, Yang H, Zhou C, Luo J, Ke ZJ. Thiamine deficiency promotes $T$ cell infiltration in experimental autoimmune encephalomyelitis: the involvement of CCL2. J Immunol 2014; 193: 2157-2167.

11. Kim RY, Hoffman AS, Itoh N, Ao Y, Spence R, Sofroniew MV, Voskuhl RR. Astrocyte CCL2 sustains immune cell infiltration in chronic experimental autoimmune encephalomyelitis. J Neuroimmunol 2014; 274: 53-61.

12. Legroux L, Arbour N. Multiple Sclerosis and T Lymphocytes: An Entangled Story. J Neuroimmune Pharmacol 2015; 10: 528-546.

13. Liu J, Gao Y, Kan BH, Zhou L. Systematic review and meta-analysis of randomized controlled trials of Chinese herbal medicine in treatment of multiple sclerosis. Zhong Xi Yi Jie He Xue Bao 2012; 10: 141-153.

14. Matsushima K, Larsen CG, DuBois GC, Oppenheim JJ. Purification and characterization of a novel monocyte chemotactic and activating factor produced by a human myelomonocytic cell line. J Exp Med 1989; 169: 1485-1490.

15. Nacka-Aleksić M, Stojić-Vukanić Z, Pilipovićl, Vujnović I, Bufan B, Dimitrijević M, Leposavić G. Strain specificities in cellular and molecular immunopathogenic mechanisms underlying development of experimental autoimmune encephalomyelitis in aged rats. Mech Ageing Dev 2017; 164: 146-163.

16. O’Brien K, Fitzgerald DC, Naiken K, Alugupalli KR, Rostami AM, Gran B. Role of the innate immune system in autoimmune inflammatory demyelination. Curr Med Chem 2008; 15: 11051115.

17. Sabatino JJ Jr, Shires J, Altman JD, Ford ML, Evavold BD. Loss of IFN-gamma enables the expansion of autoreactive CD4+ T cells to induce experimental autoimmune encephalomyelitis by a nonencephalitogenic myelin variant antigen. J Immunol 2008; 180: 4451-4457.

18. Sagar D, Lamontagne A, Foss CA, Khan ZK, Pomper MG, Jain P. Dendritic cell CNS recruitment correlates with disease severity in EAE via CCL2 chemotaxis at the blood-brain barrier through paracellular transmigration and ERK activation. J Neuroinflammation 2012; 9: 245.

19. Sharma R, Tiku AB. Emodin inhibits splenocyte proliferation and inflammation by modulating cytokine responses in a mouse model system. J Immunotoxicol 2016; 13: 20-26. 
20. Sonar SA, Shaikh S, Joshi N, Atre AN, Lal G. IFN- $\gamma$ promotes transendothelial migration of CD4+ T cells across the blood-brain barrier. Immunol Cell Biol 2017; 95: 843-853.

21. Sosa RA, Murphey C, Robinson RR, Forsthuber TG. IFN- $\gamma$ ameliorates autoimmune encephalomyelitis by limiting myelin lipid peroxidation. Proc Natl Acad Sci U S A 2015; 112: E5038-5047.

22. Sørensen TL, Tani M, Jensen J, Pierce V, Lucchinetti C, Folcik VA, Qin S, Rottman J, Sellebjerg F, Strieter RM, Frederiksen JL, Ransohoff RM. Expression of specific chemokines and chemokine receptors in the central nervous system of multiple sclerosis patients. J Clin Invest 1999; 103: 807-815.

23. Stromnes IM, Goverman JM. Active induction of experimenta allergic encephalomyelitis. Nat Protoc 2006; 1: 1810-1819.

24. Sulkowski G, Dabrowska-Bouta B, Kwiatkowska-Patzer B, Struzyńska L Alterations in glutamate transport and group I metabotropic glutamate receptors in the rat brain during acute phase of experimental autoimmune encephalomyelitis. Folia Neuropathol 2009; 47: 329-337.

25. Zhang P, Guo M, Xing Y, Yang D, Zhang L, Li Y, Xue H, Xiao B, Ma C. Immunomodulatory effect of Huangqi glycoprotein on mice with experimental autoimmune encephalomyelitis. Xi Bao Yu Fen Zi Mian Yi Xue Za Zhi 2016; 32: 54-58. 